\title{
Cerebral oxygen saturation-a useful bedside vital sign for neonatal encephalopathy
}

\author{
Sarah B. Mulkey $\mathbb{1}^{1,2,3} \cdot$ Graeme R. Polglase $\mathbb{i b}^{4}$
}

Received: 13 November 2020 / Revised: 24 November 2020 / Accepted: 14 January 2021 / Published online: 5 February 2021

(c) The Author(s), under exclusive licence to Springer Nature America, Inc. 2021

The application of near infrared spectroscopy (NIRS), a noninvasive means of monitoring cerebral oxygenation $\left(\mathrm{CrSO}_{2}\right)$, is becoming increasingly popular in many pediatric populations with the goal of identifying progression of brain injury. NIRS reflects the balance between tissue oxygen delivery and extraction. Given that the etiology of pediatric brain injury is strongly associated with abnormal cerebral perfusion and oxygenation [1], the addition of NIRS gives a more robust assessment of these variables above the standard blood pressure and oxygen saturation (pulse oximetry) measurements. Szakmar et al. in this edition of Journal of Perinatology investigated the utility of NIRS to detect improper tissue perfusion and evolving brain injury during therapeutic hypothermia and rewarming in newborn infants with mild to severe neonatal encephalopathy [2]. This retrospective study found that increased $\mathrm{CrSO}_{2}$ in the first days of postnatal life was associated with evidence of brain injury on magnetic resonance imaging (MRI), which adds to the growing literature on the potential for NIRS to be used to detect brain injury early, and potentially aid in therapeutic intervention.

NIRS has been applied in neonatal settings where cerebral perfusion and oxygenation may be affected, including ischemic encephalopathy, hypotension, apnea, patent ductus arteriosus, during extracorporeal membrane oxygenation

Sarah B. Mulkey

sbmulkey@childrensnational.org

1 Prenatal Pediatrics Institute, Children's National Hospital, Washington, DC, USA

2 Department of Pediatrics, The George Washington University School of Medicine and Health Sciences, Washington, DC, USA

3 Department of Neurology, The George Washington University School of Medicine and Health Sciences, Washington, DC, USA

4 The Ritchie Centre, Department of Obstetrics and Gynaecology, Monash University and Hudson Institute of Medical Research, Melbourne, VIC, Australia therapy, and in monitoring infants with congenital heart disease requiring surgery. Furthermore, nomograms have been developed to identify normal $\mathrm{CrSO}_{2}$ fractional oxygen extraction in neonates during the immediate transition after birth [3]. But it is really the use of NIRS in neonatal encephalopathy, which is showing the most promise, with the number of studies expanding rapidly over the past decade. Importantly, numerous studies have demonstrated that changes in cerebral oxygenation and hemodynamics are correlated with adverse neurodevelopmental outcomes and/ or brain injury [4]. Yet the role of NIRS in this setting is still very much a research tool. This likely reflects the inability of all studies to show important clinical differences between groups studied, as well as the variation in the timing of events and the relationship with adverse neurodevelopmental outcomes. For instance, Peng et al. found that differences in cerebral oxygenation in the first $12 \mathrm{~h}$ after birth were the most critical [5], while Lemmers et al. found that differences after $24 \mathrm{~h}$ of age were the critical time for adverse neurodevelopmental outcomes [6]. In the current study, Szakmar et al. found that high $\mathrm{CrSO}_{2}$ on the second day of therapeutic hypothermia was associated with gray matter injury, while high $\mathrm{CrSO}_{2}$ at the time of rewarming was associated with evidence of overall brain injury [2].

Cerebral NIRS is a trend monitor of cerebral oxygen saturation; it can have intersubject variability and does not distinguish between oxygen delivery, extraction, and cerebral blood flow, which represent inherent limitations for the tool. What is important however, is the consistency of the finding of abnormal (either high, low, or fluctuating) $\mathrm{CrSO}_{2}$ during the first days of cooling and rewarming in a patient with subsequent brain injury compared to a stable $\mathrm{CrSO}_{2}$ in a patient throughout cooling and rewarming being associated with normal outcomes, which was shown in Szakmar's study [2]. This leads to the important question of how NIRS can be incorporated into clinical practice for infants with neonatal encephalopathy. Indeed, factors including what NIRS is monitoring (cerebral blood flow, cerebral blood volume, oxygenation, or metabolism) or the use of 
NIRS in conjunction with blood pressure or an amplitudeintegrated electroencephalogram (aEEG) hinders the "easy" applicability in this setting.

The neurologic exam of an encephalopathic infant can fluctuate and be impacted by sedatives and antiepileptic medications making it difficult to follow the neurologic exam as a reliable measure of encephalopathy severity and for early evidence of recovery in some cases. Continuous neuromonitoring that could provide information about brain injury, brain recovery, and brain health and offer insight into prognosis prior to neuroimaging would improve clinical neuro-focused care in the early days of life following neonatal encephalopathy. Typically, the EEG is the only form of continuous neuromonitoring used in the NICU for infants with neonatal encephalopathy. However, the addition of cerebral NIRS as a continuous neuromonitoring tool may offer substantial benefit to the care of infants with this condition. Studies such as that by Szakmar et al. support NIRS having a prominent position at the NICU bedside [2].

The EEG, either aEEG or full-channel EEG, is a useful continuous monitor of encephalopathy and when combined with cerebral NIRS would offer a multimodal neuro vital signs approach to monitoring. EEG and cerebral NIRS are complimentary and their use together may offer the greatest benefit [7]. An infant with a static encephalopathic exam and background EEG suppression without improvement over the first few days of age during therapeutic hypothermia, combined with a trend of increasing $\mathrm{CrSO}_{2}$ on the cerebral NIRS, would be concerning for an infant with sustained cerebral injury and an abnormal neurologic outcome. However, the normalization of background EEG patterns and stable $\mathrm{CrSO}_{2}$ on NIRS may indicate a more favorable or normal neurological prognosis. This information could help guide the tone of discussions with the family for early withdrawal of care in severe cases and during the $72 \mathrm{~h}$ of therapeutic hypothermia, while families are anxiously awaiting brain MRI.

Continuous monitoring that could forewarn of clinical deterioration improves the utility of these types of tools at the NICU bedside. The HeRO (heart rate characteristics) monitor is one such bedside monitor that utilizes heart rate variability to detect an increased risk for sepsis and necrotizing enterocolitis prior to clinical signs, by a change in the continuously measured electrocardiogram signal [8]. By inclusion of a heart rate characteristics monitor, infants can have earlier laboratory evaluations and receive earlier therapeutic care to limit sepsis. This type of monitoring has been shown to reduce hospital length of stay [9]. Whether the addition of cerebral NIRS and $\mathrm{CrSO}_{2}$ monitoring could offer such benefits to the NICU population with neonatal encephalopathy is not yet known.

The NICU of the future should utilize technology to continuously analyse multiple physiological signals and help direct individualized clinical care. Mathematical models can be employed to detect warning signs in a vast array of physiological signals, including NIRS, to alert the clinical team and prompt laboratory or other neurodiagnostic assessments and establish an earlier prognosis [10]. NIRS data may be able to be applied in the future to gauge-specific treatments for neonatal encephalopathy or duration of therapy. NIRS has been vetted for several decades and despite its limitations, it has many advantages that could be used to better understand in real time the risk for neurologic injury and neurologic prognosis for infants with neonatal encephalopathy. Therefore, it is time to bring research methodologies of continuous physiological signal analysis to the bedside and this includes cerebral NIRS as an important measure of cerebral oxygenation.

\section{Compliance with ethical standards}

Conflict of interest The authors declare that they have no conflict of interest.

Publisher's note Springer Nature remains neutral with regard to jurisdictional claims in published maps and institutional affiliations.

\section{References}

1. van Bel F, Dorrepaal CA, Benders MJ, Zeeuwe PE, van de Bor M, Berger HM. Changes in cerebral hemodynamics and oxygenation in the first $24 \mathrm{~h}$ after birth asphyxia. Pediatrics. 1993;92:365-72.

2. Szakmar E, Smith J, Yang E, Volpe JJ, Inder T, El-Dib M. Association between cerebral oxygen saturation and brain injury in neonates receiving therapeutic hypothermia for neonatal encephalopathy. J Perinatol.

3. Pichler G, Binder C, Avian A, Beckenbach E, Schmolzer GM, Urlesberger B. Reference ranges for regional cerebral tissue oxygen saturation and fractional oxygen extraction in neonates during immediate transition after birth. J Pediatr. 2013;163:1558-63.

4. Mitra S, Bale G, Meek J, Tachtsidis I, Robertson NJ. Cerebral near infrared spectroscopy monitoring in term infants with hypoxic ischemic encephalopathy - a systematic review. Front Neurol. 2020;11:393. https://doi.org/10.3389/fneur.2020.00393.

5. Peng S, Boudes E, Tan X, Saint-Martin C, Shevell M, Wintermark $P$. Does near-infrared spectroscopy identify asphyxiated newborns at risk of developing brain injury during hypothermia treatment? Am J Perinatol. 2015;32:555-64.

6. Lemmers PM, Zwanenburg RJ, Benders MJ, de Vries LS, Groenendaal F, van Bel F, et al. Cerebral oxygenation and brain activity after perinatal asphyxia: does hypothermia change their prognostic value? Pediatr Res. 2013;74:180-5.

7. Goeral K, Urlesberger B, Giordano V, Kasprian G, Wagner M, Schmidt L, et al. Prediction of outcome in neonates with hypoxicischemic encephalopathy ii: role of amplitude-integrated electroencephalography and cerebral oxygen saturation measured by near-infrared spectroscopy. Neonatology. 2017;112:193-202.

8. Sullivan BA, Fairchild KD. Predictive monitoring for sepsis and necrotizing enterocolitis to prevent shock. Semin Fetal Neonatal Med. 2015;20:255-61. 
9. Swanson JR, King WE, Sinkin RA, Lake DE, Carlo WA, Schelonka RL, et al. Neonatal intensive care unit length of stay reduction by heart rate characteristics monitoring. J Pediatr. 2018;198:162-7.
10. Thewissen L, Caicedo A, Lemmers P, Van Bel F, Van Huffel S, Naulaers G. Measuring near-infrared spectroscopy derived cerebral autoregulation in neonates: from research tool toward bedside multimodal monitoring. Front Pediatr. 2018;6:117. 